\title{
DIE STÄRKE DER ERSTEN INTERNATIONALE - LEGENDE UND WIRKLICHKEIT'
}

In den Legenden ihrer Feinde wie auch ihrer Bewunderer erschien die Internationale als eine ungeheuere organisatorische und finanzielle Macht. Im dritten Prozess des französischen Kaiserreichs gegen die Mitglieder des Zentralkomitees der französischen Sektion der Internationale (8. Juni I 870), die der Geheimbündelei beschuldigt waren, behauptete der Staatsanwalt, dass die Internationale 811.5I3 Mitglieder organisiert hätte, und zwar 433.785 in Frankreich, 45.000 in der Schweiz, I 50.000 in Deutschland, 100.000 in der österreich-ungarischen Monarchie, 80.000 in England und 2.728 in Spanien. Durch diesen riesenhaften Umfang einer Organisation, die offen bekunde, dass sie ,ihr Programm nur durch eine Revolution und nur in einer demokratischen und sozialen Republik verwirklichen kann", so fügte er hinzu, sei die Internationale zu einer "sozialen Gefahr" geworden. ${ }^{2}$

Einen Monat später, am 4. Juli I $87^{\circ}$, standen in Wien vierzehn leitende Mitglieder der sozialdemokratischen Bewegung Österreichs unter der Anklage des Hochverrats vor Gericht. Der Staatsanwalt begann seine Anklagerede mit der Schilderung jenes „wichtigen Aktes, welcher für die Arbeiterbewegung der ganzen zivilisierten Welt von eminenter, gar nicht abzusehender Tragweite ist" - nämlich die Gründung der Internationale am 28. September I864. Seither

${ }_{1}$ Diese Studie ist ein Kapitel einer „Geschichte der Internationale”, deren erster Band voraussichtlich im Herbst im Verlag J. H. W. Dietz Nachf., Berlin-Hannover, erscheinen wird. Sie beruht ausser auf publizierten Quellen auf den unpublizierten Minutes of the General Council of the First International im Archiv des Internationalen Instituts für Sozialgeschichte, Amsterdam; im Text der Abhandlung wird auf diese Quelle unter dem 'Titel Protokoll der Generalratssitzung verwiesen. Der Autor wünscht, dem Direktor des Instituts, Herrn Professor Dr. A. J. C. Rüter, für die Erlaubnis zu danken, diese wichtige Quelle zu benützen. Aufrichtigen Dank schuldet er ferner den Herren Werner Blumenberg, Wissenschaftlicher Mitarbeiter des Instituts, und Leo Valiani, die das Manuskript prüften, und R. P. Morgan, der ihm seine bisher unveröffentlichte Studie The German Social Democrats and The First International zum Gebrauch zur Verfügung stellte.

${ }^{2}$ Oscar Testut, l'Association Internationale des Travailleurs (Lyon, 1870), S. 310 ; Edmond Villetard, Histoire de l'Internationale (Paris, 1872), S. 313. 
habe sich die Internationale, so führte er aus, „ganz im stillen neben den Regierungen und unter ihnen zu einer zweiten Macht im Staat, einer zweiten Regierung im Staat entwickelt, welche eine gefährliche und umso bedenklichere Konkurrenz bildet, als diese zweite Macht, diese zweite Regierung, ihre Hilfsquellen und ihre Unterstützung nicht allein in diesem Staat selbst, sondern im Ausland, und nicht vielleicht nur in einem Land des Auslandes, sondern in der ganzen Welt findet."1

Der Staatsanwalt unterliess es, diese „zweite Macht” mit Ziffern zu illustrieren. Dies unternahm wenige Wochen später die Wiener Polizeidirektion in einer Denkschrift an das Ministerium des Inneren. Nach einer ziemlich wohlunterrichteten Darstellung ihrer Entwicklungsgeschichte kommt sie zum Ergebnis, dass die Internationale „eine, wie versichert wird, dermalen über eine Million zählende, in ganz Europa und in Nordamerika verbreitete Verbindung mit revolutionärem Zweck ist."2

Die Schätzungen der Mitgliedziffern der Pariser wie auch die der Wiener Staatsbehörden wurden jedoch bald darauf von der Times, dem angesehenen Blatt des englischen Grossbürgertums korrigiert; nach ihrer Schätzung belief sich die Mitgliederzahl der Internationale auf ungefähr 2.500.000. ${ }^{3}$ Aber auch die Times scheint geirrt zu haben. Denn Oscar Testut, der sich die Erforschung der Internationale zur Lebensaufgabe gesetzt und ausser einem reichlich mit Dokumenten ausgestatteten Werk über die Internationale, ein „Blaubuch" - eine Sammlung von Dokumenten der Internationale - und eine zweibändige Studie ihrer Rolle in Europa publiziert hatte, ${ }^{4}$ kommt in seiner Schätzung ihrer Mitgliederzahl auf ungefähr 5.000.000. Uber ihre Finanzquellen konnte General Friedrich von Bernhardi „von zuverlässiger Seite" berichten, dass sie ,in London einen Barfonds von über 5.000.000 Pfund Sterling (100.000.000 Goldmark) angesammelt habe, über den sie verfügen könne."5

Soweit die Legenden der Feinde der Internationale. Die ihrer Freunde waren jedoch kaum weniger phantastisch. So berichtete dem Baseler Kongress (September I 869) Robert Applegarth, ein

${ }^{1}$ Der Wiener Hochverratsprozess (Wien, I9II), S. 648 u. 650.

2 Ludwig Brügel, Geschichte der österreichischen Sozialdemokratie, (Wien, 1922), Bd. II, S. 46 ..

3 The Times, 5. Juni 1871 .

- Oscar Testut, L'Association internationale des travailleurs (Lyon, 1870), Le livre bleu de l'Internationale (Paris, 1871 ) und L'Internationale et le jacobinisme au ban de l'Europe (Paris, 1872). Eine gekürzte Fassung seiner Bücher erschien in deutscher Übersetzung unter dem Titel: Die Internationale, Ihr Wesen und Bestrebungen (Leipzig, 1872).

5 Friedrich von Bernhardi, Tagebuchblätter aus dem Jahre 1867 bis 1869 (Leipzig, 1901), Bd. VIII, S. 406 . 
Mitglied des Generalrats, für England über einen Stand von 95.000 Mitgliedern der Internationale, in 230 Sektionen organisiert mit einem Fonds von 1.700 Pfund Sterling, J. P. Becker für die deutsche Schweiz über einen Stand von 30.000 Mitgliedern, und César de Paepe für Belgien über einen Stand von 64.000 Mitgliedern. A. C. Cameron, Schriftleiter des Workingman's Advocate in Chicago, begrüsste den Kongress als Delegierter der National Labor Union im Namen von 800.000 organisierten amerikanischen Arbeitern. L'Internationale, das Organ der belgischen Sektion der Internationale, berichtete (27. März I 870), dass die Internationale ,bereits in Europa und Amerika einige Millionen Arbeiter unter ihrer Aegide versammelt."1

Die nüchterne Wirklichkeit kontrastiert jedoch einigermassen mit diesen Legenden, vor allem mit der Legende von den märchenhaften finanziellen Hilfsquellen der Internationale.

Sie waren in Wahrheit von einer ergreifenden Dürftigkeit. Der Generalrat hatte in einer seiner ersten Sitzungen den Jahresbeitrag für individuelle Mitglieder mit I Schilling ( 1 Mark), und den für die kollektiv, durch Gewerkschaften angeschlossenen Mitglieder mit 3 Pence (etwas über 24 Pfennige) festgesetzt. Die Gewerkschaften fanden jedoch diesen Betrag zu hoch. Der Generalrat reduzierte ihn hierauf auf einen halben Penny (ungefähr 4 Pfennige). ${ }^{2}$ Aber selbst dieser Betrag schien den Gewerkschaften unerschwinglich. So begnügte sich der Generalrat mit einer jährlichen Pauschalsumme. Diese war, um sie an einigen Beispielen zu illustrieren, vom Verband der Vereinigten Tischler und Schreiner für 9000 Mitglieder 2 Pfund, von der Maurergewerkschaft mit 4000 Mitgliedern I Pfund, von der Zigarrenarbeitergewerkschaft I Pfund 9 Pence, von der Bandwebergewerkschaft von Coventry für ihre tausend Mitglieder 5 Schillinge, von einer der Buchbindergewerkschaften $x_{7}$ Schillinge 6 Pence, von der Gewerkschaft der Orgelbauer 2 Schillinge I Penny, vom Gewerkschaftsrat von Birmington I Pfund.

Jahresberichte über die Gewerkschaftsbeiträge finden sich unter

${ }^{1}$ Die Neigung der Führer von Massenbewegungen, die Zahl ihrer Anhänget zu übertreiben, um ihrer Bedeutung ein grösseres Gewicht zu verleihen, liegt wohl im Wesen der politischen Kämpfe. So bemerkte einmal Victor Adler, eine jeder Demagogie so abholde Natur, zu Scheidemann: „Wissen Sie, mit den Millionen bin ich nie kleinlich gewesen. Ich hab' mehr als einmal im Namen von ein paat Millionen gesprochen, wenn auch bloss ein paar hundert Leute hinter mir gestanden sind". Philipp Scheidemann, Der Zusammenbruch (Berlin, 1921), S. 125 .

2 Protokoll der Generalratssitzung, 9. September 1866. 
den Dokumenten der Internationale nicht; wohl aber fand sich ein vom Schatzmeister Cowell Stepney bearbeiteter Bericht über das Einkommen des Generalrats während der ersten sechs Jahre aus Beiträgen der individuellen Mitglieder. Es ergaben sich folgende Summen: 1865 - 23 Pfund; 1866 - 9 Pfund I 3 Schillinge; 1867 5 Pfund 17 Schillinge; 1868 - 14 Pfund 14 Schillinge; 1869 - 30 Pfund I 2 Schillinge; I 870 - I4 Pfund I4 Schillinge. Der letzte, von Engels dem Haager Kongress vorgelegte Finanzbericht für die Jahre 1870-1872 wies ein Defizit von "über 25 Pfund" aus, ein Betrag, den der Generalrat „einigen seiner Mitgliedern und anderen” schuldete. ${ }^{1}$ Das Gesamteinkommen des Generalrats für das Finanzjahr I869-1 $870 \mathrm{zum}$ Beispiel betrug 5 I Pfund 7 Schillinge I Penny, die Ausgaben während dieses Jahres 47 Pfund 7 Schillinge 5 Pence, aber der noch unbeglichene Rückstand an Mietzins 4 Pfund ${ }_{4}$ Pence.

Der Mietzins und das Gehalt des Generalsekretärs verursachten dem Generalrat nicht geringe Sorgen. Der Mietzins betrug 4 Schillinge (4 Mark) in der Woche; er konnte trotz wiederholter Mahnungen und Drohungen mit gerichtlicher Delogierung kaum jemals voll bezahlt werden. „Der Generalrat schuldet 5 Wochen Miete und schuldet seinem Sekretär", klagte Marx in einem Brief an Engels (22. Juli I 869). ${ }^{2}$ Das Gehalt des Sekretärs kam erst nach dem Genfer Kongress (September 1866) zur Sprache, da bis dahin George Cremer als ehrenamtlicher Sekretär kein Gehalt bezog (er hatte ein Einkommen als Sekretär der Bauarbeitergewerkschaft). Marx beantragte im Generalrat, das Gehalt seines Nachfolgers mit 2 Pfund die Woche festzusetzen; der Generalrat bewilligte jedoch nur I Pfund. Aber selbst für dieses bescheidene Gehalt war das Geld häufig nicht vorhanden. So reduzierte der Generalrat (Ende Mai I 867) das Gehalt auf den Betrag von to Schillingen 6 Pence, und beschloss, das Geld durch eine Sammlung unter den jeweils den Sitzungen beiwohnenden Mitgliedern des Generalrats aufzubringen. Diese Sammlungen ergaben in der ersten Woche I I Schillinge; in den folgenden Wochen: 9 Schillinge; 8 Schillinge; 7 Schillinge; 7 Schillinge 6 Pence; nichts; 6 Schillinge; nichts; 3 Schillinge; und schliesslich 8 Schillinge, worauf diese Finanzaktion eingestellt wurde. Und da es immer schwieriger wurde, selbst den Betrag von to Schillingen 6 Pence für den Generalsekretär der Internationale aufzubringen, beantragte John Hales (I I Oktober 1870), ihn auf s Schillinge die Woche herabzusetzen (der Antrag wurde allerdings mit 6 Stimmen gegen eine verworfen). Mit welchen Summen der Generalrat zu rechnen hatte, illustrieren

\footnotetext{
${ }^{1}$ Siehe The First International - Minutes of the Hague Congress of 1872 with related documents, hrg. Hans Gerth (Madison, 1958), S. 223.

${ }^{2}$ Marx-Engels Briefwechsel, Bd. IV, 2. S. 25 I.
} 
zum Beispiel die folgenden Eintragungen in die Sitzungsprotokolle: „Der Generalsekretär wird ermächtigt, den Betrag von 6 Pence für Briefmarken seiner Korrespondenz in Angelegenheit einer Solidaritätsaktion in Deutschland für die streikenden Schneider in London auszulegen” (27. März I 866); oder: „Der Sekretär wird ermächtigt, ein Kassabuch zu kaufen" (2. Februar i 869). Marx scherzte kaum, als er in einem vertraulichen Brief dem Ausschuss der sozialdemokratischen Partei Deutschlands (24. März I870) mitteilte, „dass die Finanzen des Generalrats unter dem Nullpunkt sind, beständig wachsende negative Grössen". ${ }^{1}$ General Bernhardi wie selbst Appelgarth hatten die finanziellen Hilfsquellen der Internationale einigermassen überschätzt.

Ungleich schwieriger ist es jedoch, den Wahrheitskern aus den Legenden von der gewaltigen organisierten Heeresmacht der Internationale herauszuschälen. Statistische Aufzeichnungen über den Stand der Mitglieder der Internationale sind nicht vorhanden. Die Zahl ihrer individuellen Mitglieder war unzweifelhaft gering. In England zum Beispiel betrug sie bis Ende I 870 nicht mehr als 294. In Frankreich und in der Schweiz war ihre Zahl zwar erheblich grösser, aber es können insgesamt nur einige Tausende gewesen sein. So berichtet zum Beispiel das Pariser Zentralkomitee von einem Stand von nur 36 Sektionen in Frankreich im Jahre $1870 .^{2}$

In Deutschland schien es, als wäre die Werbung von individuellen Mitgliedern der Internationale besonders schwierig. Liebknecht, so klagte Marx in einem Brief an Engels (5. August 1865), ,hat es nicht fertig gebracht, für die International Association auch nur eine Branche von 6 Mann in Deutschland zu bilden". ${ }^{3}$ August Bebel wurde erst 1867 Mitglied der Internationale. Erfolgreicher war Beckers Werbearbeit für die Internationale in Deutschland, die er von Genf in der Schweiz unternahm. Johann Philipp Becker (I 809I886), ein Revolutionär in Temperament und Geisteshaltung, hatte als Freischarenkommandant in der Revolution von 1848 hervorragend gekämpft, und wurde später ein begeisterter Anhänger der Ideen von Garibaldi wie Lassalles, und von Marx wie Bakunins. Ende 1865 gründete er in Genf die Monatschrift Der Vorbote, die

1 Der Hochverrats-Prozess wider Liebknecht, Bebel, Hepner ((Berlin, 1894), S. 337.

2 Protokoll der Generalratssitzung, I9 Juli 1870; E. Dolléans, Histoire du mouvement ouvrier, Bd. I, I 830-1871 (Paris, 1936); Y. M. Stekloff, History of the First International (London, 1928 ).

${ }^{3}$ Marx-Engels Briefwechsel, Bd. III, S. 337. 
of the venom of impotent hatred; he speaks with deep emotion of their passing: "Evil war and awful battle slew them all". 1

Many are the stories: of Jason's Argonauts and the fearful revenge of Medea, of the siege of Troy and the fall of ash-speared Priam's folk, of the wanderings of the returning heroes and the bloody aftermaths of their homecomings - all these we know. They mark the breakup of the age of bronze, but the iron has not yet struck. Cherished gauds and trinkets, and sometimes an uncanny tool or weapon, give warrant for the saying, "Iron doth of itself draw a man on" 2, but the strange gray metal is not yet a common thing. ${ }^{3}$ The earlier Homeric Epic, and all that surrounds it, is still a tale of the struggles of the men of bronze against each other, not against the men of iron. The chilled edges have yet to slash through bronze helms and corselets, to pierce

1 Ibid.

2 Od. xvi, 294; xix, 13.

3 "Gray" iron, od. ix, 393; "flaming" iron, od. i, 184; iron axes, Il. xxiii, 850; "selfsmelted" or "self-poured" - i.e., probably metcoric iron (but see John L. Myres, Who Were the Greeks?, Vol. VI: Sather Classical Lectures [Berkeley: The University of California Press, 1930], p. 442, and J. A. Rickard, "The Primitive Smelting of Iron", American Journal of Archeology, XLIII, No. I [January-March, 1939], pp. 85-86); Il .xxiii, 826; iron knife, Il. xviii, 34; iron mace or club - wielded by a hero whose skill with this most unusual weapon brought him fame, Il. vii, I4I; iron etters, Od. i, 204; iron gates - of the nether world, and hence perhaps not thought of as an ordinary metal, Il. vii, 15; iron chariot axle - of the goddess Hera, hence probably "precious" iron, Il. v, 623.

Iron is mentioned, counting adjectival and compound forms (Alban Dawes Winspear, The Genesis of Plato's Thought [New York: The Dryden Press, Inc., I940], p. 313 , note 10), 22 times in the Iliad, 25 in the Odyssey. This leads Winspear to say, "Iron is mentioned slightly more often in the Odyssey than in the Iliad" (The Genesis of Plato's Thought, p. 20). This is correct enough, but what Winspear fails to take full account of is the proportional mention; on this basis iron is mentioned four times as often in the Odyssey. Let us give these proportions: in the Iliad and Odyssey combined, iron accounts for almost 9 per cent of the references; in the Iliad alone, slightly more than 5 per cent; in the Odyssey alone, a trifle more than 20 per cent.

Note, now, that even if one were disposed to grant Myres' contention (Who Were the Greeks?, p. 435), that from bronze to iron "the transition is now known to have been gradual, and to have been more rapid in some districts than in others" - and the present writer is certainly disposed to grant it, for "ages" do not start on schedule - it is still true that the Iliad, generally acknowledged to be the earlier part of the Homeric Epic (see end of note 5), grants little place to iron except as a rare or even precious metal. Bronze is mentioned almost twenty times as often. Cf. Fritz Taeger, Das Altertum (4th ed.; Stuttgart: W. Kohlhammer, 1950), p. I 2.

For further discussions of iron in Homer, see Martin P. Milsson, Homer and Mycenae (London: Methuen and Co., Ltd. 1933), p. 139 and the articles and books he cites in footnote 5; Arnold J. Toynbee, A Study of History (2nd ed.; London: Humphrey Milford, 1935), III, p. 16r; A. R. Burn, Minoans, Philistines and Greeks (New York: Alfred A. Knopf, 1930), pp. 226-27; Robin Léon, Greek Thought and the Origins of the Scientific Spirit (New York: Alfred A. Knopf, 1928), p. 75 and footnote I; E. C. Semple, The Geography of the Mediterranean Region (New York: Henry Holt and Co., 193I), pp. 684-85. 
Dennoch gelang es der Internationale, zunächst im Süden Italiens Wurzeln zu schlagen. Im Mai 1866 kündigte die Italienische Arbeiterassoziation in Neapel ihren Beitritt zur Internationale an; sie zählte I 869, wie der Generalrat dem Baseler Kongress berichtete, ungefähr 600 Mitglieder. Aber es war erst die Pariser Commune, die den Anstoss zur Ausbreitung der Internationale in Italien gab. Die Commune wurde von den italienischen Arbeitern begeistert begrüsst, von Mazzini jedoch leidenschattlich verdammt. Garibaldi hingegen bekannte sich zu ihr und stellte ihr seine Dienste zur Verfügung, und der revolutionäre Flügel der Mazzinischen Arbeiterorganisationen vereinigte sich unter der Führung von Ludovico Nabruzzi und Erminio Pescatori auf dem Kongress in Bologna im Dezember I $87 \mathrm{I}$ in einer Gegenorganisation, Il Fascio Operaio. Bald danach entstanden Sektionen der Internationale in Bologna, Turin, Mailand, Ravenna, Faenza. Benoit Malon, ein Mitglied der Pariser Sektion der Internationale vor ihrer Unterdrückung nach dem Fall der Commune, der als ein führender Communard flüchten musste und sich in Italien niederliess, spricht von der Gründung von ungefähr hundert internationalen und revolutionären Gruppen. ${ }^{1}$ Die Gesamtzahl ihrer Mitglieder im Jahre I871 wurde offiziell mit ro.000 angegeben. Cesare Lombrose berechnet sie jedoch nur auf 2.000.

Der Kontrast dieser Ziffern erklärt sich aus der eigenartigen sozialen Struktur der Bewegung. Leonida Bissolati charakterisiert die Internationale in Italien in jener Zeit als eine Gruppierung von Gelehrten, Entgleisten, Dichtern, Enthusiasten, Altruisten und wilden Männern, ohne bestimmte Marschroute und scharf formulierte Ziele, aber durch tiefgehende revolutionäre Empfindungen und Instinkte verbunden, weniger als wirkliche besondere Organisation wie als Sammelpunkt verschiedenartiger Kräfte und Kampfgenossenschaften zu ethischen Zwecken. Robert Michels, der Bissolatis Bemerkung in seiner Studie zitiert, lässt dies mit Einschränkungen nur für die Führerschaft der Internationale gelten; die Masse ihrer Mitglieder habe sich aus dem städtischen und ländlichen Proletariat rekrutiert. Aber auch das Proletariat in Italien war damals noch nicht fähig, stabile Organisationen zu entwickeln. Unter dem Antrieb aufwühlender Ereignisse oder hinreissender Redner strömten sie in die Sektionen der Internationale, aber verliefen sich nach einiger Zeit.

In Spanien entwickelte sich eine unabhängige sozialistische Bewegung erst nach der spanischen Revolution im September 1868.

\footnotetext{
'Benoit Malon, Il Socialismo, suo Passato, suo Presente e suo Avenire (Lodi, I875), zitiert in Robert Michels, Proletariat und Bourgeoisie in der sozialistischen Bewegung Italiens, im Archiv für Sozialwissenschaft und Sozialpolitik, Bd. XXI, S. 358; ferner Richard Hostetter, The Italian Socialist Movement I860-1880 (Princeton, 1958), Bd. I.
} 
Der Generalrat der Internationale erliess einen Monat später eine Proklamation an die „Arbeiter Spaniens”, und die Sozialisten, die bis dahin einen Flügel der Republikanischen Partei gebildet hatten, lösten sich von ihr und schufen selbständige Arbeiterorganisationen. Sie standen vorwiegend unter anarchistischer Führung. Im März 1869 konstituierte sich in Barcelona, bald darauf in Madrid eine Sektion der Internationale, und in der revolutionären Atmosphäre Spaniens in diesen Jahren breitete sie sich reissend aus, vor allem in Catalonien und Andalusien. Farga-Pellicer, einer der beiden Delegierten zum Baseler Kongress (September I 869), berichtete für ganz Spanien von einem Stand von 195 Sektionen mit ungefähr 20.000 Mitgliedern der Internationale, unter ihnen 38 Sektionen mit 708 I Mitgliedern in Barcelona. Engels allerdings behauptete, wie er dem Generalrat berichtete, dass die Bewegung durch innere Kämpfe zwischen Anarchisten und Marxisten, und wohl auch durch Regierungsverfolgungen gelähmt wäre. ${ }^{1}$

In den Vereinigten Staaten entstanden 1868 zunächst deutsche, bald darauf auch englische, französische, tschechische, irische und skandinavische Sektionen der Internationale, und zwar in New York, Chicago, San Francisco, New Orleans, Newark, Springfield, Washington und Williamsburg - insgesamt 30 Sektionen mit ungefähr 5.000 Mitgliedern im Jahre I 87 I. $^{2}$ Aber im folgenden Jahr spaltete sich die nordamerikanische Föderation der Internationale im Hader zwischen ihren proletarischen und intellektuellen Mitgliedern, und auf ihrem Kongress im Juli 1872 waren nur mehr 22 Sektionen mit insgesamt 950 Mitgliedern vertreten - und zwar 12 deutsche, 4 französische, 3 englisch-sprechende und je eine irische, italienische und skandinavische. ${ }^{3}$

Diese Übersicht über den Stand der individuellen Mitglieder der Internationale ergibt sich aus einer kritischen Prüfung der den Sitzungen des Generalrats vorliegenden sehr dürftigen Berichte der nationalen Sektionen wie auch aus anderen Quellen.

\section{IV}

Aber für die Schätzung der Grösse der Bewegung der Internationale ist es wichtig, festzuhalten, dass die individuellen Mitglieder nur eine der beiden Gruppen der Gesamtorganisation der Internationale bildeten. Ein ungleich stärkere Gruppe bildeten die durch Gewerk-

${ }^{1}$ Protokoll der Generalratssitzung, 3 Januar 1870.

2 Morris Hilquit Geschichte des Sozialismus in den Vereinigten Staaten (Stuttgart I906), S. I 85 .

${ }^{3}$ Hermann Schlüter, Die Internationale in Amerika (Chicago, 1918), S. 177. 
schaften und politische Vereine organisierten kollektiven Mitglieder.

Der Generalrat sah in der Gewinnung der englischen Gewerkschaften eine seiner Hauptaufgaben. ${ }^{1}$ Er entsendete Delegationen zu Gewerkschaftsvorständen, um sie zum Anschluss an die Internationale zu bewegen oder zumindestens um ihre Sympathien für sie zu gewinnen. Es war eine bedeutende Errungenschaft des Generalrats, dass der Kongress der englischen Gewerkschaften in Sheffield I 866 in einer einstimmig beschlossenen Resolution der Internationale „für ihre Bemühungen, die Arbeiter aller Länder durch ein gemeinsames Band der Brüderlichkeit zu vereinigen, volle Anerkennung" aussprach und ,allen auf dem Kongress vertretenen Gewerkschaften den Anschluss an die Internationale" empfahl. Als Robert Applegarth dem Baseler Kongress (1 869) von einem Stand von 95.000 Mitgliedern der Internationale in England berichtete, waren in der Tat bereits 28 Gewerkschaften angeschlossen, unter ihnen die Gewerkschaft der Mechaniker mit 33.000 Mitgliedern und die der Erdarbeiter mit 28.000 Mitgliedern.

Nicht selten kam es zu spontanen Massenanschlüssen an die Internationale im Feuer schwerer Gewerkschaftskämpfe. Eine der konkreten Aufgaben, die die englischen Gewerkschaftsführer der Internationale bei ihrer Gründung zugewiesen hatten, war die Verhinderung von Streikbrüchen durch importierte ausländische Arbeiter und die Organisation von Solidaritätsaktionen für streikende Arbeiter. Der Generalrat war im Grunde eine Vertretung der englischen Gewerkschaftsbewegung. Die Hälfte seiner Mitglieder waren englische Gewerkschaftsführer, sein Präsident, George Odger, war gleichzeitig Generalsekretär des Londoner Gewerkschaftsrates, und die Sekretäre und Schatzmeister der Internationale waren führende Männer in der englischen Gewerkschaftsbewegung, so auch der deutsche Schneider Georg Eccarius. Nahezu in jeder Sitzung des Generalrates standen Gewerkschaftskämpfe zur Beratung - Zuschriften oder Delegationen streikender Gewerkschaften, die die Internationale aufforderten, durch ihre Organisation die Einfuhr ausländischer Streikbrecher abzuwehren oder streikende Arbeiter durch Geldsammlungen zu unterstützen.

Der Generalrat intervenierte in der Tat in zahllosen Streiks, die durch ausländische Streikbrecher bedroht waren; so zum Beispiel in den Streiks der Londoner Drahtarbeiter, der Schneider von Edinburgh, der Londoner Tabakarbeiter, der Schneider in Manchester, der Londoner Korbflechter, der Londoner Schneider. Er appellierte durch seine Sektionen und durch Arbeiterblätter in Frankreich,

\footnotetext{
${ }^{1}$ Siehe George Howell, Labour Movements and Labour Leaders (London, 1902); A. W. Humphrey, Robert Applegarth (London, 1913).
} 
Belgien, Holland und Deutschland an die Solidarität der von den Agenten englischer Unternehmer angeworbenen Arbeiter, die häufig nicht wussten, dass ihnen die Rolle von Streikbrechern zugedacht war, und bewegte sie in der Regel, das englische Arbeitsangebot abzulehnen. „Eine der Hauptanklagepunkte im Prozess gegen die Leiter der Pariser Sektion der Internationale war", berichtete der Generalrat in einem Rundschreiben (Juli 1869) an die englischen Gewerkschaften, „dass sie während des Streiks der englischen Zinkarbeiter, Schneider und Eisenbahnangestellten die französischen Arbeiter vor der Abreise nach England zurückgehalten haben."

Diese Akte hilfreicher Solidarität trugen den Ruhm der Internationale in tausende Arbeiterfamilien. Die meisten der streikenden Arbeiter hatten wohl zum ersten Mal in Streikversammlungen und Zeitungsberichten vom Dasein eines Bruderbundes der Arbeiter erfahren, der die Macht besass, durch Aktionen in Frankreich, Holland und Belgien Kämpfe der Arbeiter in Manchester, Edinburgh und London zu unterstützen. Die natürliche Reaktion auf dieses Erlebnis war, dass sie sich diesem Bruderbund als zugehörig fühlten.

Das Ansehen der Internationale wuchs ins legendarische, als bekannt wurde, dass es ihr da und dort gelungen war, Kämpfe der Arbeiter durch ihr Eingreifen zu einem erfolgreichen Abschluss zu bringen.

Die Reihe dieser Aktionen begann mit einer Intervention der Internationale im Kampf der Pariser Bronzarbeiter im Februar 1867. Die Unternehmer hatten ungefähr I.500 Arbeiter ausgesperrt und weitere 4.000 mit Aussperrung bedroht, weil sie sich weigerten, ihre kurz vorher gegründete Gewerkschaft aufzugeben. Die ausgesperrten Arbeiter riefen die Hilfe der Internationale an. Der Generalrat wendete sich an den Londoner Gewerkschaftsrat und an die Sektionen der Internationale auf dem Kontinent um finanziellen Beistand. Kleinste wie grössere Beträge strömten ein: zum Beispiel ro Pfund von den Londoner Buchbindern, 20 Pfund von der Londoner Sektion der Tischler, 5 Pfund von der englischen Tabakarbeitergewerkschaft, jedoch auch 4.000 Pfund aus einer Sammlung der französischen Gewerkschaften und eine Anleihe von 10.000 Pfund von der englischen Hutarbeitergewerkschaft. Es war dies die erste finanzielle Solidaritätsaktion der Internationale, und ihre Wirkung auf die Arbeiter wie auf die Unternehmer war, wie Fribourg, ein Mitglied des Zentralkomitees der Pariser Sektion der Internationale in seinem Buch $^{1}$ erzählt, überwältigend. Sie stärkte das Selbstbewusstsein der Arbeiter und ihre Zuversicht und verblüffte die Unternehmer. Diese gaben einige Tage nach dem Eintreffen grösserer Geldsendungen aus

${ }^{1}$ E. E. Fribourg, L'association internationale des travailleurs (Paris, $187 x$ ), S. ror. 
London ihre Forderungen auf und öffneten ihre Werkstätten. Die Arbeiter schlossen sich in die Hunderte der Pariser Sektion der Internationale an.

Im Streik der Genfer Bauarbeiter (Frühling I 868) für eine Verkürzung der Arbeitszeit von I 2 auf ro Stunden genügte allein das Gerücht, dass der Generalrat in London den Bauarbeitern in Genf ein Darlehen von 40.00o Frank im Monat für die Dauer des Streiks zugesichert hätte, die Unternehmer zu Verhandlungen und zu einem Kompromiss zu bewegen. Der Streik, der die ganze Stadt erregte, hatte sich zu einem Kampf zwischen dem Bürgertum und der Internationale zugespitzt, und die Internationale hatte ihn gewonnen. In jenen Tagen schlossen sich der Genfer Sektion etwa tausend Mitglieder an.

Eine ähnliche Wirkung in ungleich grösserem Ausmasse hatte die Intervention des Generalrates im Streik der Seidenspinner von Lyon. "Trotz polizeilicher Einschüchterung erklärten die Arbeiter öffentlich ihren Anschluss an die Internationale", berichtete der Generalrat dem Baseler Kongress, „andere Arbeitergruppen folgten ihrem Beispiel und wir gewannen mehr als I0.000 neue Mitglieder".

Die Kunde von erfolgreichen Interventionen der Internationale verbreitete sich in vielen Ländern. Unter den Appellen um Beistand in Arbeiterkämpfen, die die Protokolle der Generalratssitzungen verzeichnen, finden sich die der Spinner von Rouen, der Schriftsetzer von Leipzig, der Pariser Eisengiesser (um eine Streikanleihe, die ihnen durch die Intervention des Generalrates von der englischen Gewerkschaft der Mechaniker gewährt wurde), der Papierarbeiter von New York (die den Generalrat aufforderten, den Export europäischer Lohndrücker zu verhindern), der Weber von Vienne in Frankreich, der deutschen wie auch später der belgischen Tabakarbeiter, der Sozialdemokratischen Partei Deutschlands (um eine Anleihe für die streikenden Bergarbeiter von Waldenburg).

Der Generalrat verfügte, wie wir gesehen haben, über keine eigenen Geldmittel. Selbst die Reserven der Gewerkschaften waren in jener Zeit noch dürftig, und die Sektionen der Internationale schwach und arm. Jeder Hilferuf um Beistand stellte den Generalrat vor ein Problem. Einen Einblick in die finanziellen Hilfsquellen der Internationale für Streikunterstützungen gewährt zum Beispiel der Bericht über eine Geldsammlung für ungefähr 800 ausgesperrte Seidenfärber und Bandweber in Basel, der der Sitzung des Generalrates am 9. März I 869 vorlag. Die Aussperrung war erfolgt, weil sich diese Gruppe der Arbeiter zum Entsetzen der guten Bürger dieser Stadt öffentlich der Internationale angeschlossen hatten. Sie appellierten 
an den Generalrat und der Generalrat an die Sektionen der Internationale. Die erste Sammelliste ergab ungefähr 300 Pfund. Unter den Beiträgen fanden sich folgende: vom Generalrat - 4 Pfund; von der Londoner Webergewerkschaft - I Pfund; vom deutschen Kommunistischen Arbeiterbildungsverein in London - 3 Pfund 7 Schillinge; von der Genfer Sektion der Internationale - 30 Franken; von einer öffentlichen Versammlung in Paris - 135 Franken; von einer öffentlichen Versammlung in Basel - 62 Franken. Es wurde ferner berichtet, dass die Bewohner von zwei Dörfern in der Nachbarschaft von Basel den ausgesperrten Arbeitern Kartoffeln, Äpfel, Gemüse und Brennholz geschickt hatten. Es sei noch hinzugefügt, dass die ausgesperrten Arbeiter durchhielten und die Unternehmer sich mit der Zugehörigkeit der Arbeiter zur Internationale abzufinden hatten.

$\mathrm{Zu}$ einem Massenanschluss an die Internationale kam es in Belgien nach den blutigen Zusammenstössen zwischen Bergarbeitern und Truppen im Bergwerkrevier von Charleroi. Die Kohlenbarone hatten im Frühling I 869 unter dem Druck einer Wirtschaftskrise die Arbeitszeit in den Gruben auf vier Tage in der Woche beschränkt und gleichzeitig die Löhne um ro Prozent herabgesetzt. Die Bergarbeiter protestierten in Streiks und Strassendemonstrationen. In Charleroi schritt Militär ein, schoss auf die Demonstranten, tötete elf und jagte selbst die Verwundeten in den Strassen. Viele Arbeiter wurden nachher verhaftet, unter ihnen auch die leitenden Mitglieder der belgischen Sektion der Internationale. Der Generalrat erliess (am 4. Mai I 869) ein von Marx verfasstes Manifest, „An die Arbeiter von Europa und den Vereinigten Staaten", das die belgische Regierung in leidenschaftlicher Sprache vor aller Welt anklagte. ${ }^{1}$ Gleichzeitig organisierte er Sammlungen für die Opfer des Kampfes und Rechtsbeistand für die Verhafteten. Die Ereignisse im Kohlenrevier hatten die Arbeiterschaft des ganzen Landes aufgewühlt. Sie schlossen sich in die Tausende der Internationale an. Rudolf Meyer behauptete, dass sich im Gefolge der Streiks in Frankreich - der Streiks der Baumwollarbeiter in Rouen, der Seidenspinner in Lyon und der Kohlenarbeiter in St. Etienne - in den Jahren I868 und 1869 die Zahl der Mitglieder der Internationale um mehr als 50.000 erhöht hatte. ${ }^{2}$

Massen, die in der Erregung ihrer Kämpfe der Internationale zu-

\footnotetext{
${ }^{1}$ Für den Wortlaut des Manifests siehe Karl Marx und Friedrich Engels über die Gewerkschaften (Berlin, 1953), S. I 2 ff.

${ }^{2}$ Rudolf Meyer, Der Emanzipationskampf des vierten Standes (Berlin, I874), Bd. I, S. 122. Meyers zweibändiges Werk ist die umfassendste dokumentarische Darstellung der Geschichte der Ersten Internationale und ihrer Sektionen in deutscher Sprache. Siehe auch Gustav Jaeckh, Die Internationale (Leipzig, I 904).
} 
strömten, verliefen sich jedoch zum grössten Teil nach einiger Zeit. Wohl aber verblieb das Gefühl der Zugehörigkeit zu diesem grossen internationalen Arbeiterbund, der ihnen in der Stunde der Not beigestanden hatte. Auch der Beitritt der Gewerkschaften zur Internationale war häufig nur ein Ausdruck ihrer Gefühle der Solidarität mit ihren Klassengenossen anderer Nationen.

Auf einer einigermassen festeren Grundlage beruhten die Beziehungen zwischen sozialistischen Parteien und der Internationale. Aber zur Zeit der Ersten Internationale gab es nur in Deutschland und Österreich Ansätze einer sozialistischen Reichspartei; in Deutschland der von Ferdinand Lasalle 1863 gegründete Allgemeine deutscher Arbeiterverein und der von Liebknecht und Bebel geführte Verband deutscher Arbeitervereine, aus dem auf dem Kongress in Eisenach (August I 869) die Sozialdemokratische Partei Deutschlands hervorging. Keine der beiden Parteien schloss sich der Internationale formell an, weil, wie es z.B. Bebel auf dem Eisenacher Kongress begründete, „sich zunächst die sozialdemokratische Partei in Deutschland selbst konstituieren müsse, da neben der internationalen auch die nationale Organisation unentbehrlich ist und jene ohne diese nur ein Schatten wäre."1 Aber beide Parteien erklärten sich mit der Internationale solidarisch. Das vom Eisenacher Kongress beschlossene Parteiprogramm übernahm nahezu im Wortlaut die Prinzipienerklärung in den Statuten der Internationale und erklärte, dass sich die Partei, ,soweit es die Vereingesetze gestatten, als Zweig der Internationalen Arbeiterassoziation betrachtet". Überdies empfahl der Kongress den Parteimitgliedern den persönlichen Beitritt zur Internationale.

In ähnlicher Weise behandelte die österreichische sozialdemokratische Bewegung, die mit der Gründung des Wiener Arbeiterbildungsvereines im November 1867 ins Dasein trat und bald einen Massenanhang gewann, die Frage ihrer Beziehung zur Internationale. Die österreichische Regierung hatte "die Bestrebungen" der Internationale als „staatsgefährlich" erklärt; eine offene organisatorische Verbindung mit der Internationale stand unter der Strafsanktion des Hochverrats. Die Partei konnte daher formell der Internationale nicht beitreten. Aber sie handelte in ihrem Geiste. Das erkannte natürlich auch die Regierung, als sie im Juli 1870 vierzehn leitende

'Mehring, ebd. Bd. III, S. 368 . Für eine Diskussion der taktischen Gründe, die sowohl Schweitzer wie Liebknecht bestimmten, eine abwartende Haltung gegenüber der Internationale einzunehmen, siehe Morgan, ebd. 
Mitglieder der Partei unter der Anklage des Verbrechens des Hochverrates vor Gericht stellte. Die Anklageschrift konnte zwar eine organisatorische Verbindung der Partei mit der Internationale nicht erweisen, aber sie stützte ihre Beschuldigung auf die Tatsache, dass Andreas Scheu und Heinrich Oberwinder (die Hauptangeklagten) „als Mandatträger von nahezu 100.000 Oesterreichern" den Eisenacher Kongress begrüsst und in ihren Versammlungsreden und Zeitungsartikeln bekundet hatten, dass sie mit „den Ideen und Grundsätzen [des Eisenacher Programms, das die Grundsätze der Internationale übernommen hatte] nicht nur vollkommen sympathisieren, sondern auch alles daran setzen, um dieselben zu realisieren". ${ }^{1}$ Und da die Angeklagten dies keineswegs bestritten, wurden sie wegen des Verbrechens des Hochverrats zu einigen Jahren schweren Kerkers verurteilt.

Obgleich die österreichische Partei der Internationale nicht angeschlossen, und die Zahl jener, die ihr als Mitglieder beigetreten waren, überaus gering, und wohl auch die Zahl von „100.000 Genossen", in deren Namen Scheu und Oberwinder den Eisenacher Kongress begrüssten, wahrscheinlich reichlich übertrieben war, so konnte dennoch geschlossen werden, dass sich grosse Massen der österreichischen Arbeiterklasse mit der Internationale solidarisch verbunden fühlten. In einem „Exposé”, das der Ministerialrat SchmidtZabierow für eine von den Regierungen der österreich-ungarischen Monarchie und Deutschlands zur Beratung von Massnahmen gegen die Internationale einberufene Konferenz im Sommer 1872 vorbereitet hatte, wird angenommen, dass in Wien ,aktionsbereit 20.000 Arbeiter als Anhänger der Internationale oder vielmehr der sozialdemokratischen Partei zu zählen sein dürften, während die noch nicht disziplinierten Arbeitermassen zirka I 50.000 betragen."2

Der Kontrast zwischen der geringen Zahl der individuellen Mitglieder der Ersten Internationale und dem Ausmass des Einflusses auf die Gefühlswelt der Arbeiter trat besonders eindrucksvoll in Frankreich, in Paris, Lyon, Marseille und anderen grösseren Städten in Erscheinung. Die französischen Sektionen der Internationale waren kaum mehr als Zellenorganisationen, manche allerdings einige hundert Mitglieder stark. Aber durch den Einfluss ihrer individuellen Mitglieder in den Gewerkschaften, Genossenschaften und anderen Arbeiterorganisationen und politischen Klubs, und begünstigt durch die revolutionäre Stimmung in der Arbeiterklasse, wurde die Internationale in Frankreich, wie auch in Belgien und Italien Ende der sechziger Jahre eine Massenbewegung.

${ }^{1}$ Der Wiener Hochverratsprozess, S. 278-279.

${ }^{2}$ Für das „Exposé” vom 8. Juni 1872 siehe Brügel, ebd., Bd. II, S. I45. 
Ein symptomatisches Beispiel für die Ausstrahlungen des Einflusses der Internationale demonstrierte auch eine Episode in der Geschichte der amerikanischen Arbeiterbewegung. Die Sektionen der Internationale in den Vereinigten Staaten vereinigten, wie wir zeigten, in der Zeit ihrer kurzen Blüte nur einige tausend Mitglieder (unter ihnen, wie Marx dem Generalrat mitteilte, Wendel Phillips, der grosse Vorkämpfer der Sklavenbefreiung ${ }^{1}$ ). Aber es gelang ihnen, die Sympathien der National Labor Union - der Nationalen Arbeiterunion - zu gewinnen, einer Organisation von mehr als sechzig Gewerkschaften, die auf einem Kongress in Baltimore im Jahre I866 unter der Führung von William $\mathrm{H}$. Sylvis gegründet worden war. Der Generalrat lud sie im Mai r 869 ein, eine Delegation zu dem bevorstehenden Baseler Kongress der Internationale zu entsenden. Der Kongress der Nationalen Arbeiterunion (Philadelphia, August I 869) nahm die Einladung an und wählte A. E. Cameron als Delegierten, der, wie bemerkt, den Baseler Kongress im Namen von 800.000 amerikanischen Arbeitern begrüsste. Und schon ein Jahr später erklärte die Nationale Arbeiterunion (auf ihrem Kongress in Cincinnati, August I 870), ihre Übereinstimmung mit den Prinzipien der Internationalen Arbeiterassoziation" und kündigte an, dass sie ihr "in kurzem beitreten" würde. ${ }^{2}$ Dazu kam es freilich nicht, weil sie einer Apathie in der Arbeiterklasse zum Opfer fiel, die durch eine Reihe schwerer Niederlagen in Massenstreiks in den Jahren I 871 und 1872 ausgelöst worden war.

Aber der Niedergang der amerikanischen Arbeiterbewegung verminderte anscheinend nicht das öffentliche Interesse an der Internationale. Ein charakteristisches Zeugnis für ihr Ansehen bot die Debatte im Repräsentantenhaus des Kongresses der Vereinigten Staaten (I 5 . Dezember 187 I) über die Einsetzung einer Kommission zur Erforschung der Lage der Arbeiter. Der Gesetzentwurf war vom republikanischen Kongressmitglied R. Hoar, später Generalstaatsanwalt im Kabinett Cleveland eingebracht worden. In seiner Rede zur Begründung der Vorlage verlieh er ihr ein besonderes Gewicht durch den Hinweis, dass ,die grosse Internationale Arbeiterassoziation, eine Organisation”, wie er sagte, „die sich über ganz Europa erstreckt und die ihrer Stimme überall Gehör verschafft und ihre Macht in allen Kreisen fühlbar macht, den Kongress der Vereinigten Staaten um die vorliegende Massregel ersucht habe". Er fügte hinzu, dass „die internationale Assoziation europäischer und amerikanischer Arbeiter unter anderem schon deshalb Anspruch auf Achtung hat, weil sie die Nationen der Welt einander nahe

1 Protokoll der Generalratssitzung, 15 . August 1871 .

2 Hilquit, ebd., S. 181. 
gebracht hat, weil sie eine Verwandtschaft zwischen Menschen und Menschen anerkennt, eine Verwandtschaft, die dem gemeinsamen Bande der Arbeit entspringt". Und er erinnerte ,an die düstersten Tage unseres eigenen Krieges”, als die „herrschenden Klassen Englands und der Kaiser von Frankreich" die amerikanischen Nordstaaten mit einer Intervention bedrohten, die nur „das zornige Grollen der Arbeiter von Lancashire verhütete". Schliesslich liess er durch den Sekretär des Repräsentantenhauses die Beschlüsse der Londoner Delegiertenkonferenz der Internationale vom September I 87 I und des Genfer Kongresses von 1866 verlesen, die eine statistische Aufnahme der Lage der arbeitenden Klassen aller Länder anregten. ${ }^{1}$ Es sei hinzugefügt, dass das Repräsentantenhaus, das der Rede Hoar's wiederholt Beifall zollte, den Gesetzentwurf annahm und der Senat zwar der Einsetzung einer Untersuchungskommission zustimmte, jedoch die Entscheidung über die im Entwurf geforderte Einrichtung eines nationalen statistischen Büros vertagte.

So weit die überprüfbaren Data über die Mitgliederbewegung der Internationale und des Ausmasses ihres Einflusses. Sie ergeben kein abgerundetes Bild. Der Versuch, die Bewegung statistisch zu erfassen, scheitert an der Dürftigkeit der Quellen. Aber sie lassen jedenfalls erkennen, dass die Berichte von einer straff organisierten Millionenarmee der Internationale, dirigiert von ihrem Londoner Generalrat, in den Bereich der Legende gehören. Die Internationale kommandierte nicht organisierte Massen. Sie war, wie die Londoner Times einmal schrieb, „ein grosser Gedanke in einem kleinen Körper”. Aber es gelang ihr, in grossen Arbeitermassen Gefühle der Solidarität mit der Internationale zu erwecken und den Respekt vieler fortschrittlichen Intellektuellen und Sozialreformer zu gewinnen. Darauf beruhte ihre von den herrschenden Klassen so gefürchtete Stärke.

1 Schlüter, ebd., S. 144-145. 